\title{
GLOBALIZACIÓN, INTEGRACIÓN REGIONAL Y DESARROLLO COOPERATIVO: ANÁLISIS DEL CASO CHILENO
}

\section{GLOBALIZATION, REGIONAL INT E GRATION AND COOPERATIVE DEVELOPMENT : ANALYSIS OF CHILEAN CASE}

\section{RESUMEN}

LUIS HERNÁNDEZ ASTUDILLO

\author{
Programa Interdisciplinario de Estudios Asociativos Pro - Asocia \\ Universidad de Chile \\ E-mail: luhernan@uchile.cl \\ MARIO RADRIGÁN RUBIO \\ Programa Interdisciplinario de Estudios Asociativos Pro - Asocia \\ Universidad de Chile \\ E-mail: mradriga@uchile.cl
}

\begin{abstract}
El sector cooperativo a nivel internacional, al igual que otras formas de empresas, se ha visto sometido en los últimos 25 años a lo menos, a los efectos crecientes del proceso de globalización, frente al cual se han ensayado diversas respuestas. El presente trabajo es un esfuerzo de realizar un análisis del caso concreto del sector cooperativo chileno, y de sus esfuerzos y estrategias de actuación en el contexto de la globalización y de los procesos de integración comercial. Este trabajo es un resultado parcial del proyecto "Impacto de la integración regional del MERCOSUR sobre el sector cooperativo", financiado por el Centre de Recherche pour le Dévelopement International, CRDI, del Canadá, y que ha coordinado la Universidad de la República del Uruguay.
\end{abstract}

Palabras Claves: Desarrollo Cooperativo, Integración Regional, Globalización, Comercio Internacional.

\begin{abstract}
This work represents an effort to analyze the particular case of the Chilean cooperative sector, and specially his efforts and strategies to conduce their cooperatives in the context of the globalization and the commercial integration process. This document is a partial result of "Regional Integration and the Cooperative Sector (MERCOSUR)" project, financed by the Centre de Recherche pour le Development International, CRDI, of Canada, and coordinated by the "Universidad de la República del Uruguay
\end{abstract}

Key Word: Cooperative Development, Globalization, International Trade, Regional Integration. 


\section{1.- INTRODUCCIÓN.}

Si bien es cierto que desde hace siglos los contactos comerciales e intercambios de todo tipo de ideas, productos, innovaciones y rasgos culturales, se han dado de forma frecuente entre las distintas regiones del planeta, y de ellos son testimonio el desarrollo de los diferentes tipos de "imperios" a nivel mundial desde hace más de 4.000 años, nunca antes en la historia de la humanidad este tipo de contactos habían sido tan acelerados ni habían tenido la capacidad de hacer empequeñecer al planeta, hasta transformarla en una "aldea global". Este es el fenómeno que actualmente conocemos como "globalización". La globalización es un fenómeno que conlleva múltiples variables: sociales, culturales, políticas, militares, tecnológicas, histórico-geográficas, entre otras. Tiene en la actualidad también un impacto profundo en los diversos procesos de integración comercial y regional que se potencian en las diversas regiones del mundo: ALCA, UE, MERCOSUR, Comunidad Andina, ASEAN, por mencionar sólo algunas.

Por su parte, el modelo cooperativo moderno, que tiene más de 150 años de trayectoria, debe su origen a las consecuencias inmediatas de un fenómeno que está vinculado a los inicios del proceso de globalización actual, como lo fue la Revolución Industrial, y que entre otras cosas fue una respuesta de los sectores y clases más desfavorecidas, para mejorar sus condiciones de vida. Una parte importante del desarrollo cooperativo se impulsó en contextos de diversas políticas proteccionistas de las economías nacionales, que en la actualidad o han entrado en crisis o tienden a desmontarse, lo que ha significado en muchos casos severas crisis, y la necesidad de aj ustes dramáticos.

I caso chileno es particularmente ilustrativo de los agudos procesos que se viven en el marco de la globalización, y en su relación con el sector cooperativo nacional. Chile inicia en pleno período del Régimen Militar un proceso acelerado de liberación económica a finales de los años '70, coincidiendo con un desmantelamiento de las políticas de apoyo al sector cooperativo, y en algunos casos un hostigamiento directo. El modelo económico chileno, de orientación neoliberal se consolida en la década de los '90, ya recuperadas las instituciones democráticas, habiendo firmado tratados de libre comercio con Canadá, México y Estados Unidos, además de con la Unión Europea, China y Corea, y avanzadas negociaciones con India y J apón.

En este período el sector cooperativo ha detenido su descenso, y comienza un lento crecimiento, si bien no en el número de empresas cooperativas, si en su número de socios/as, teniendo que actualizar de forma drástica sus estrategias de gestión comercial, para enfrentar un entorno cada vez más complejo y competitivo. En este trabajo, se presenta este proceso y prefigura sus tendencias de futuro.

\section{2.- LA GLOBALIZACIÓN: PROCESOS Y TENDENCIAS ACTUALES}

\section{1. - La globalización algo más que una palabra y un proceso}

La globalización es quizá uno de los fenómenos de mayor análisis, debate y difusión de los últimos 25 años, sin embargo el concepto de globalización no tiene una sola definición ni enfoque, y es objeto en la actualidad de una profunda reflexión a nivel mundial, que en algunos casos dice relación con distintos conceptos que tienen semejanzas y diferencias entre sí, tales mundialización, globalización, internacionalización. A su vez, desde un punto de vista práctico y que afecta a la totalidad de los países del planeta, la 
globalización tiene una relación directa con los procesos de integración regional e integración comercial

Se ha insistido más de una vez que el proceso de globalización es un fenómeno multidimensional y de creciente complejidad, y la mayor parte de los autores clásicos que abordan el fenómeno de la globalización se centran o dan énfasis a uno de sus ámbitos o dimensiones.

Por ejemplo existe la posibilidad de analizar los procesos de globalización desde una perspectiva histórica: la globalización como ciclos largos y ciclos cortos, en el cual se constata a su vez una aceleración de los procesos históricos.

Pero también se puede enfocar la globalización desde una perspectiva política, haciendo referencia a planteamientos tales como el fin de la historia y la aldea global. También es posible identificar una perspectiva humanista-social, en donde las preocupaciones sobre la extensión y la la difusión de la democracia y los derechos humanos tiene una gran fuerza. Existe otra opción que corresponde a la formulación de planteamientos propios de una perspectiva tecnológica-informacional, apuntando hacia la sociedad de la información o informacional.

Por otra parte, el enfoque económico-comercial, que plantea la opción un mundo sin fronteras ni barreras, el cual tiene un gran peso en por sobre los otros enfoques. La perspectiva comunicacional y de los medios de masas tiene en la actualidad gran impacto y refleja la sensación de un mundo cada vez más pequeño

Finalmente el enfoque o perspectiva cultural guarda relación con las preocupaciones que relacionan ala cultura global, las tradiciones locales y la industria cultural.

\section{2. - Las diversas valoraciones de la globalización}

Complementariamente a los enfoques o ámbitos con los cuales se puede analizar la globalización, se puede hacer también una síntesis general sobre las diversas valorizaciones que tiene la globalización para distintos actores y agentes a nivel internacional, nacional y local, tales como el que plantea que esta consiste en la culminación de la civilización, dando cuenta de la aparición de nuevos etnocentrismos y el síndrome del progreso ilimitado (por lo menos para algunos).

Para otros autores la globalización es como puerta abierta, y que por ende hay un mundo de posibilidades abiertas para quienes puedan utilizarlas. Sin embargo otros pensadores y especialmente los movimientos sociales plantean la demonización de la globalización, en donde esta es vista como madre de todos los males y los movimientos antiglobalización.

\section{3. - Debates sobre la "gestión" o administración de la globalización}

Otra dimensión de fuerte y gran debate actual tiene que ver con las distintas perspectivas para evaluar cuales son los grados o posibilidades de "gestión" del proceso de globalización, y si esto fuese posible o necesario, donde recaería esta responsabilidad.

Para algunos es el "mercado" es el principal piloto de la globalización, lo que corresponde a una nueva versión postmoderna del laisse-faire, para otros, corresponde que sea el sistema multinacional y multilateral (ONU, OMC, FMI, etc.) los que debieran ser los 
garantes del proceso. Por otra parte desde los movimientos sociales, se plantea una propuesta de relevar el rol de la sociedad civil en los procesos de mundialización. Y finalmente para otros aún ven en los Estados-Nación actores relevantes incluso en los actuales procesos de globalización.

\section{4. - Globalización, integración regional y su impacto sobre las estrategias de gestión y desarrollo de las empresas cooperativas}

Tal como ya se ha señalado en los puntos anteriores, el sector cooperativo a nivel internacional se encuentra sometido en la actualidad, a uno de sus mayores desafíos de toda su historia, como lo es enfrentar un escenario de desarrollo económico y social, marcado por los efectos del proceso de globalización.

Para las empresas cooperativas ya no es posible enfrentar sus procesos de desarrollo y crecimiento pensando tan solo en sus entornos locales y nacionales, pues cotidianamente en sus diversos sectores de actividad, se ven enfrentados a diversos embates de la globalización, que tienen que ver tanto con los fenómenos de la circulación del capital como con el desmontaje de los diversos mecanismos que, por décadas generaron los estados nacionales para proteger sus economías de la competencia exterior.

Si bien es cierto que los diversos bloques económicos, y especialmente la Unión Europea y los Estados Unidos, mantienen una serie de restricciones a la libre competencia a nivel internacional, como por ejemplo barreras para-arancelarias, cuotas y controles sanitarios, como también las propias prácticas de la Política Agrícola Común, PAC, en la Unión Europea, y las diversas trabas que la OMC no ha podido desbloquear, asistimos a un nivel creciente del comercio internacional, a la deslocalización de las empresas y a un competencia cada vez más fuerte, que no pueden dejar al sector cooperativo como simples espectadores.

Existen diversas formas de analizar la relación entre globalización, integración regional y su impacto sobre las estrategias de gestión y desarrollo cooperativo. Por lo tanto en esta sección entregaremos una visión específicamente desde dos ángulos. El primero de ellos relativo al discurso de los propios actores del sector cooperativo, especialmente desde una óptica latinoamericana, para en segundo término realizar identificar las líneas de investigación académica y aplicada sobre el tema, con especial atención a los impactos de los procesos generales de la globalización sobre el sector cooperativo.

\section{5. - Globalización y sector cooperativo desde la perspectiva de los actores cooperativos}

Desde la perspectiva de los propios actores, tanto en base a la revisión documental, sus propias declaraciones como entrevistas procesadas a través de estudios de caso, podemos encontrar las siguientes cuatro perspectivas generales, que nos permiten aproximarnos al análisis de la relación entre desarrollo cooperativo y globalización e integración regional, desde la óptica de los propios actores de las empresas cooperativas (dirigentes y ejecutivos). Es claro que estas visiones son una aproximación y simplificación de los diversos discursos posibles de identificar, y que efectivamente pueden existir diversas combinaciones entre ellos. 
- El enfoque declarativo - poético: el cooperativismo como alternativa de humanización de la globalización, pero sin resultados concretos

- El enfoque pragmático: si no puedes vencerlo únete a ellos, o los negocios son los negocios

- El enfoque de trinchera: la cooperativa como factor de resistencia a la globalización

- El enfoque de la intercooperación globalizada: incipiente pero creciente. El comercio justo

\section{6. - Globalización, integración regional y sector cooperativo desde la perspectiva de la investigación académica y aplicada}

\subsection{1- Identificación general de líneas de investigación desarrolladas}

Sin querer ser exhaustivos ni menos dar cuenta de la totalidad de líneas de trabajo e investigación en el campo de la globalización, integración regional y comercial y las estrategias de gestión de las cooperativas, a continuación se presenta de forma esquemática una serie de líneas de estudios que dan cuenta de diversas investigaciones realizados 0 en curso que dicen relación directa con la relación entre globalización y gestión y desarrollo del sector cooperativo, en donde, en más de un caso se producen cruces y relaciones de mutua imbricación, en función de las preocupaciones específicos y de los marcos teóricos que cada investigador y autor ocupa para impulsar su trabajo.
a) Procesos de deslocalización y externalización productivo de las empresas cooperativas
b) La constitución de holdings y grupos cooperativos
c) Empresas cooperativas, globalización y precarización de los empleos
d) Contrapuntos entre la identidad local y las actuaciones globales
e) La desmutualización y privatización de las cooperativas
f) La gobernabilidad de las empresas cooperativas globales
g) Las actuaciones comerciales de las cooperativas en la economía global
h) Políticas públicas, marcos legales y actuación de las cooperativas en los contextos globales
i) Procesos de integración regional y comercial y su impacto sobre el sector cooperativo
j) Análisis globales ideológico/teóricos
k) Desarrollo cooperativo, globalización y comercio justo
I) Cooperación al desarrollo, globalización y cooperativismo 


\subsection{2.- Tipos de cooperativas actuantes en marcados globalizados}

Específicamente en el campo de las empresas cooperativas que han asumido la opción de su internacionalización de forma plena y directa, tema que plantea múltiples dudas, cuestionamientos y desafíos, especialmente en lo que dice relación a sus desnaturalización y perdida de apego a los principios cooperativos clásicos, para pasar a constituirse en una transnacional más, existe en la actualidad una tipología de empresas transnacionales, que permiten identificar diversos procesos y consecuencias, tanto en las áreas de gestión propiamente comercial, y también en los temas del gobierno corporativo y sus impactos sobre la democracia y participación societal.

De este particular tipo de cooperativas transnacionales, y siguiendo a Novkovic (2006), podemos identificar tres tipos particulares de cooperativas:

- Cooperativas locales vinculadas a redes globales

- Cooperativas que se han creado expresamente para actuar en mercados globales y que tienen asociados en diversos países

- Cooperativas locales que se han transformado en cooperativas con una implantación global

\subsection{3.- Desafíos para la gestión de cooperativas en marcados globalizados}

- El desafío de lograr entender con claridad lo que está pasando con la globalización:

- El desafío de evaluar como posicionar su actividad empresarial en el contexto de mercados globales

- El desafío de conciliar los principios cooperativos con la Internacionalización de la empresa cooperativa

- El desafío de explorar formas innovadoras de intercooperación a nivel internacional

- El desafío de generar fórmulas de gobierno corporativo democráticas y participativas a nivel internacional

- El desafío de establecer alianzas estratégicas con otros actores sociales y económicos públicos y privados.

\section{3.- ESTRATEGIA GLOBAL DE DESARROLLO DE CHILE.}

A partir de la segunda mitad de la década de los años '70, y una vez ya definido el modelo económico - social a instaurar por parte del régimen militar del Gral. Augusto Pinochet $U$., se comienza a configurar en Chile la estrategia global de desarrollo que con algunos matices, permanece hasta la actualidad. Una estrategia marcadamente aperturista y orientada hacia los mercados internacionales.

Tal como señala Furche (2001), probablemente los dos aspectos centrales y característicos del Ilamado "caso chileno", son: 
- Es mejor dedicarse a producir aquellos bienes donde se tienen ventajas comparativas o se están desarrollando ventajas competitivas y adquirir los demás bienes y servicios a quienes lo producen más barato (otros países),

- El reducido tamaño de la economía chilena, lleva a la necesidad de ampliar el mercado externo, el cual se convierte en elemento fundamental de la estrategia de desarrollo nacional.

El desarrollo de esta estrategia, ha involucrado la aplicación de diversas políticas, donde cabe resaltar al menos las dos principales: el regionalismo abierto, y, la política de fomento de las exportaciones. ${ }^{1}$

En primer lugar, el denominado "regionalismo abierto", es decir, la preferencia por la vinculación con países de la región versus otros, más alejados, manteniendo abierta la posibilidad de negociar acuerdos en cualquier momento con cualquier país del mundo. Esta política comercial, iniciada en la década de los '90, ha tenido importantes éxitos y se ha caracterizado por la búsqueda de negociaciones comerciales con diferentes países para ampliar su acceso a nuevos mercados de exportación. La relación con estos países se ha regulado a través de convenios internacionales o bilaterales, en los que la certificación en origen y el reconocimiento mutuo, son sus características principales.

En este contexto, y en el ámbito multilateral, Chile ha sido miembro desde su origen del Acuerdo General sobre Aranceles Aduaneros y Comercio (GATT), participa en la Organización Mundial para el Comercio (OMC), participa del Acuerdo sobre el Sistema Global de Preferencias Comerciales entre Países en Desarrollo (SGPC), hace parte de grandes proyectos de integración comercial en la Región (Acuerdo de Libre Comercio de las Américas ALCA, Asociación Latinoamericana de Integración ALADI, Mercado Común del Sur MERCOSUR), y desde el año 1985 forma parte del Foro de Cooperación Económica del Asia Pacifico APEC, entre otros.

Por su parte, y esta vez en el ámbito del "libre comercio", podemos destacar al menos los siguientes acuerdos / tratados:

- Acuerdos a Asociación Económica: Singapur, Nueva Zelanda y Brunei (firmado en 2005).

- Tratados de Libre Comercio: Australia, Vietnam, Malasia y Tailandia (2007, inicio de negociaciones); Perú (2006, inicio de negociaciones); Colombia (2005, inicio de negociaciones); J apón (2005, inicio de negociaciones); Panamá (firmado en 2006); China (firmado en 2005); Corea del Sur (firmado en 2003); Estados Unidos de América (firmado en 2003); Lienchtenstein, Noruega, Suiza e Islandia (firmado en 2003); El Salvador, Nicaragua, Honduras, Costa Rica y Guatemala (firmado en 1999); México (firmado en 1998); Canadá (firmado en 1996).

- Acuerdos de Alcance Parcial: India (firmado en 2005); Unión Europea (firmado en 2002).

- Acuerdo de Complementación Económica: Perú (firmado en 1998), Argentina, Brasil, Uruguay y Paraguay (firmado en 1996); Ecuador (firmado en 1994); Bolivia (firmado en 1993); Colombia (firmado en 1993); Venezuela (firmado en 1993).

Así entonces, el país en el tema de acuerdos comerciales ha mantenido una postura flexible entre el enfoque multilateral (OMC) y los acuerdos bilaterales.

Por otra parte, y en segundo término, en el decenio de los noventa las autoridades chilenas centraron sus esfuerzos de fomento de las exportaciones, en la utilización de instrumentos que apuntaban a corregir directamente algunas distorsiones del mercado. El

\footnotetext{
${ }^{1}$ En este apartado, nos basamos en el análisis desarrollo por Luis Lobo G. (2007).
} 
principal programa de este conjunto de herramientas, administrado por el ente nacional encargado del fomento de las exportaciones (Dirección de Promoción de Exportaciones ProChile, dependiente del Ministerio de Relaciones Exteriores), se encargó de suministrar principalmente información a los potenciales exportadores y apoyo en las actividades de promoción de productos nacionales en nuevos mercados. Estas políticas tuvieron éxito en facilitar el acceso al mercado a las empresas participantes.

La liberalización comercial chilena es la más antigua y de aplicación más persistente en América Latina. A finales de 1973, se inició una audaz reforma de la política comercial que comprendió la eliminación de todas las restricciones no arancelarias, un abrupto proceso de reducción de los aranceles y la unificación de los tipos de cambio múltiple en una tasa única.

También es importante mencionar que el notable desempeño de las exportaciones chilenas ha estado vinculado durante las últimas dos décadas a políticas "heterodoxas" más activas, que procuraron preservar un tipo de cambio real competitivo y generar una capacidad exportadora, en lugar de limitarse únicamente a implantar reformas económicas "ortodoxas", como ocurrió en los años setenta con la liberalización comercial unilateral. Ricardo French - Davis (2002), identifica cuatro factores, que se retroalimentan recíprocamente para explicar este desempeño de las exportaciones chilenas:

- Tipo de cambio real depreciado: lo cual constituye una variable determinante de la trayectoria global de las exportaciones. Aunque, en los últimos años, este tipo de cambio se ha apreciado de una forma importante debido a la situación de debilidad del dólar a nivel mundial y el propio éxito del modelo exportador que ha presionado la apreciación del tipo de cambio.

- Mejoramiento de la calidad de las exportaciones, basada en una diversificación en favor de bienes y servicios con mayor valor agregado.

- Acentuado esfuerzo nacional dirigido a completar los mercados internos de tecnología y de capacitación laboral y los segmentos de largo plazo de los mercados de capitales, que en su conjunto permitió elevar la calidad de las exportaciones.

- Entorno macroeconómico interno sustentable y en el que prevalecieron precios macroeconómicos claves, que no estaban desalineados y que fueron adecuados para

el desarrollo productivo que facilitó el impulso exportador.

En definitiva, la expansión dinámica de las exportaciones ha sido un rasgo distintivo de la economía chilena en el último cuarto de siglo. Las exportaciones de bienes y servicios han mostrado una tendencia ascendente como proporción del PIB y elevándose desde el $15 \%$ en los setenta, a cerca del $30 \%$ a principios del actual decenio (Lobo, 2007). Sin embargo también existen criticas a este modelo en el cual la exportación y la atención constante a la evolución de la demanda en los mercados extranjeros, se ha convertido en uno de los principales componentes de la estrategia país, dejando de lado el desarrollo del mercado interno y excluyendo a aquellas empresas que no son capaces de acceder al mercado internacional. Situación esta, en la que se encuentran la mayor parte de las pequeñas y medianas empresas, incluidas por cierto las cooperativas, y que representan casi el $80 \%$ del empleo nacional.

Otra importante debilidad del modelo chileno, es el cuestionable impacto que tiene el éxito exportador en el desarrollo social y económico del conjunto del país, fundamentalmente en la distribución de la riqueza generada. Así, del total exportado en el país durante el año 2002, el $50 \%$ había sido embarcado por 25 empresas (Lobo, 2007). La mayoría de estas empresas se dedican a la extracción y exportación de recursos naturales, entre minería, agricultura, pesca y madera o derivados directos de estos recursos 
("primarización de las exportaciones"). Se trata en general de grandes empresas, es decir, existe una alta concentración empresarial exportadora, así del $60 \%$ de las empresas representan apenas el $0,4 \%$ del total exportado durante el año 2002. Además de las 25 empresas que representan el $50 \%$ del valor exportado por Chile, 13 de ellas pertenecen a capital extranjero (Lobo, 2007). Adicionalmente, no es posible evidenciar una relación directa entre el crecimiento de las exportaciones y del producto interno bruto; al respecto French - Davis (2002), al caracterizar el "modesto crecimiento medio del PIB", indica que el crecimiento anual promedio del volumen de las exportaciones, de un $10 \%$ entre los años 1974 y 2001, convivió para el mismo período de tiempo, con un apenas 4,3\% de crecimiento promedio del producto interno bruto.

Finalmente, y a pesar de los buenos indicadores, la economía de Chile aún presenta un grave defecto: la desigual distribución de ingresos entre la población, lo que genera una gran brecha social entre ricos y pobres. Según el Informe de Desarrollo Humano de la ONU en 2005, Chile se encuentra en el puesto 113 de la lista de países por igualdad de ingreso, ubicándose entre las 15 últimas naciones a nivel mundial, lo que revela una importante carencia en la economía que aún no ha podido ser subsanada.

\section{4.- ANTECEDENTES GENERALES DEL SECTOR COOPERATIVO CHILENO.}

Si bien es cierto, que la historia nacional consigna una serie de antecedentes de organizaciones "pre-cooperativas", existe consenso en cuanto a situar la primera organización propiamente "cooperativa" de Chile, en el año 1887, con la constitución en la Ciudad de Valparaíso de la Sociedad Cooperativa de Consumo La Esmeralda (Del Campo y Radrigán; 1998). El surgimiento del cooperativismo en Chile tiene directa relación con el movimiento social y obrero de fines del siglo XIX, así como también la densificación urbana y los inicios de la industrialización del trabajo especialmente dado por las compañías salitreras del norte grande del país. Desde sus inicios a la fecha el movimiento cooperativo en Chile ha pasado por varias etapas, períodos de florecimiento y otros de decaimiento especialmente influenciados por el contexto socio político del país.

El inicio del proceso de "transición a la democracia", tras los resultados del Plebiscito del año 1988, significó para vastos sectores de la población la generación de una gran cantidad de expectativas, muchas de las cuales no pudieron ser satisfechas inmediatamente, y otras definitivamente no han sido asumidas hasta la fecha. Para el sector cooperativo y de la economía social en general, existía la expectativa de un cambio más profundo del modelo económico neoliberal impulsando por el Régimen Militar a contar del año 1975, o incluso su reemplazo. Se pensaba que las nuevas autoridades iban a tener una mayor consideración por propuestas alternativas de organización económica, como es por ejemplo el modelo cooperativo, sin embargo hasta ahora, eso no ha podido concretarse.

A pesar de esta cierta indiferencia gubernamental, el sector cooperativo chileno, progresiva y lentamente desde 1990 en adelante, y con no pocos "pasos en falso", ha venido generando interesantes -aunque también incipientes- procesos de adaptación al nuevo contexto nacional e internacional.

Desde el año 1990, y hasta el año 2004 (incluido), el Departamento de Cooperativas del Ministerio de Economía², registra la creación de 503 nuevas cooperativas. Tal como es

\footnotetext{
2 El Departamento de Cooperativas es la repartición pública en Chile, a cargo del fomento, supervisión y fiscalización de las Cooperativas.
} 
posible observar en el Gráfico $\mathrm{N}^{\circ} 1$, dentro de ese período es el año 1992, donde se alcanza el mayor número de cooperativas creadas, alcanzando a 56.

\section{Gráfico N 1.}

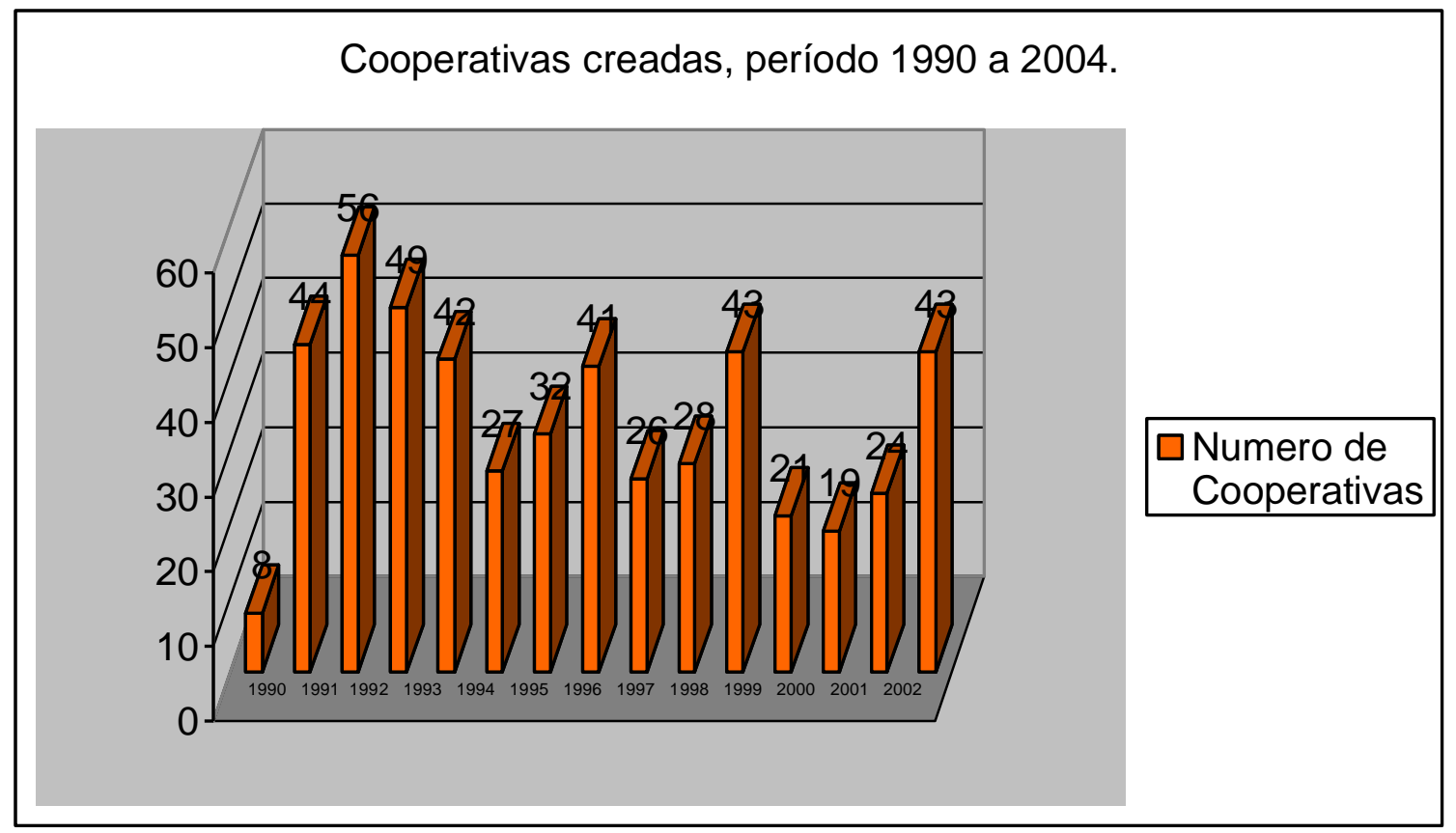

Fuente: Hernández (2006), a partir de datos del Departamento de Cooperativas del Ministerio de Economía.

De acuerdo a estimaciones del propio Departamento de Cooperativas (2007 a), actualmente las cooperativas en Chile generan 36.679 puestos de trabajo. Por su parte, y tal como se muestra en el Cuadro $N^{\circ} 1$, al 31 de Octubre de 2004, el número de socios/ as de cooperativas en Chile alcanzó a 1.255.712.-, representando un 8,3 \% de la población total del país, uno de los porcentajes más altos en la historia (Castillo et al.; 2006). Estas cifras, están directamente relacionadas con el explosivo aumento de miembros de cooperativas de ahorro y crédito, que en los últimos diez años han crecido en más de un $400 \%$ Ilegando hoy a representar casi un $60 \%$ del total de chilenos/as asociados/as a cooperativas. $^{3}$

Cabe consignar respecto de los datos estadísticos de cooperativas en Chile, que éstos muestran algunas importantes falencias en cuanto a representatividad y actualización, puesto que por ejemplo el concepto de "cooperativas activas", hace referencia a aquellas cooperativas que han presentado algún tipo de documentación (balance, actas o similares) al Departamento de Cooperativas en los últimos cinco años. ${ }^{4}$ Dichas debilidades tienden a revertirse con la incorporación de las cooperativas en el

${ }^{3}$ Tan sólo la Cooperativa de Ahorro y Crédito del Personal de la Universidad de Chile COOPEUCH Ltda., muestra a la fecha más de 370.000 socios/ as. Según el Ranking "Las CAC's más grandes de América Latina", elaborado por la Confederación Alemana de Cooperativas DGRV, COOPEUCH es la segunda Cooperativa de Ahorro y Crédito más grande de América Latina y el Caribe (después de la Caja Popular Mexicana).

${ }^{4}$ Por oposición entonces, las "inactivas" son aquellas cooperativas de las cuales el Departamento no ha tenido ninguna información durante los últimos cinco años, y que sin embargo no han sido formalmente disueltas (forzada o voluntariamente). 
cuestionario de la Encuesta Nacional de Empleo que aplica el Instituto Nacional de Estadísticas INE -acogiendo las recomendaciones en ese sentido de la Organización Internacional del Trabajo OIT-, y la actual realización del Primer Catastro Nacional de Cooperativas, por parte del Departamento de Cooperativas en el marco del Programa de Fortalecimiento para la Regulación y Supervisión del Sector Cooperativo (BID / FOMIN).

A continuación se presenta un cuadro resumen, con la última información disponible (al 31 de Octubre de 2004).

\section{Cuadro $\mathbf{N}^{\circ} 1$.}

\begin{tabular}{|l|r|r|r|}
\cline { 2 - 4 } \multicolumn{1}{c|}{ Tipo de Cooperativa } & \multicolumn{3}{c|}{ Cooperativas vigentes al 31 de octubre de 2004 } \\
\hline Agrícola & 60 & Inactivas & $\mathbf{N}^{\circ}$ de socios \\
\hline Campesina & 199 & 124 & 28.806 \\
\hline Consumo & 14 & 210 & 29.621 \\
\hline Minera/Pesquera & 24 & 80 & 176.768 \\
\hline Producción/Trabajo & 84 & 44 & 3.344 \\
\hline Ahorro & 84 & 48 & 4.624 \\
\hline Vivienda Abierta & 8 & 18 & 734.222 \\
\hline Vivienda Cerrada & 169 & 2 & 43.953 \\
\hline Veraneo & 41 & 561 & 36.485 \\
\hline Agua Potable & 124 & 8 & 3.198 \\
\hline Eléctricas & 19 & 23 & 53.143 \\
\hline Otros servicios & 78 & 2 & 73.678 \\
\hline Org.Integración & 17 & 68 & 67.396 \\
\hline Total & $\mathbf{9 2 1}$ & 23 & 474 \\
\hline
\end{tabular}

Fuente: Hernández (2006), a partir de datos del Departamento de Cooperativas del Ministerio de Economía.

En términos de cantidad de socios/as, junto con la ya mencionada muy importante participación del sector de ahorro y crédito, destacan también el sector consumo -con más de 170 mil socios/ as-, y las cooperativas eléctricas con cerca de 74 mil socios/ as. Entre los tres sub-sectores, representan un 78,4\% del total de cooperados/ as en Chile.

Por otra parte, y tal como queda reflejado en el Cuadro precedente, son muchas más las cooperativas de las cuáles no se dispone información en los últimos cinco años (1.211), que las cooperativas de las cuales el Departamento, ha recibido información en ese mismo período de tiempo (921).

\section{1. - Marco Normativo}

La actual Ley General de Cooperativas № 19.832 promulgada el 23 de Octubre del $2002,{ }^{5}$ ha implicado un cambio sustantivo al marco jurídico en que se desenvuelven las cooperativas chilenas en comparación a la legislación vigente hasta ese momento, que en lo sustancial databa del año 1978. Esta "nueva" Ley, tiene entre sus principios generales tres fundamentos centrales, a saber: las cooperativas generan riqueza, las cooperativas pueden competir en todos los mercados y la ley simplifica los procedimientos y trámites para el desarrollo de éstas. Este marco jurídico busca posibilitar la inserción de las cooperativas en el actual contexto económico y la adopción libre, de estrategias y

\footnotetext{
${ }^{5}$ Su entrada en vigencia es a contar de mayo del año 2003.
} 
conceptos empresariales para progresar económicamente, flexibilizando una serie de barreras burocráticas que obstaculizaban la creación y desarrollo de las cooperativas.

La nueva normativa, entre otras modificaciones, elimina el concepto según el cual las cooperativas son entes sin fines de lucro, liberalizando la repartición de excedentes entre los socios/as, facilita la obtención de personalidad jurídica para las nuevas cooperativas que se constituyan, asimilando su constitución a la de las sociedades comerciales, es decir, mediante escritura pública cuyo extracto debe inscribirse en el Registro de Comercio y publicarse en el Diario Oficial (Matamala; 2004).

Otro punto es la eliminación de las limitaciones en cuanto al objeto social, estableciéndose plena libertad para desarrollar bajo esta forma de organización, cualquier actividad económica, sin perjuicio de la regulación establecida por leyes especiales, permitiéndose además la combinación de finalidades de diversas clases de cooperativas, con la excepción de aquellas que tienen objeto único (ahorro y crédito, y vivienda abierta). Se establecen además, nuevos mecanismos de resolución de conflictos por la vía de arbitraje, en los que tendrán participación las propias Federaciones o Confederaciones. Así mismo, se autoriza expresamente la fusión, la división de las cooperativas, y su transformación en otro tipo de sociedades. Con esto se facilita la rapidez en la toma de decisiones, permitiendo una mayor competitividad de las cooperativas.

También se establece y regula el derecho a retiro para los socios/as disidentes, especialmente en caso de que se adopten decisiones de gran relevancia, como reforma del objeto social, fusión, división, transformación, entre otras. La Ley permite el ingreso al mercado de Agencias de Cooperativas Extranjeras que deseen realizar operaciones en Chile. Se radican las facultades de regulación y de supervisión del sector cooperativo en el Departamento de Cooperativas del Ministerio de Economía.

\section{5.- REFLEXIONES Y PROPUESTAS DESDE EL CASO CHILENO.}

En el contexto previamente expuesto, las cooperativas, así como el conjunto de las empresas en Chile, han debido alinearse con la estrategia de desarrollo del país. Ello, en algunos casos ha significado finalmente la desaparición de muchas cooperativas, la apenas "supervivencia" de otras, así como también en algunos casos, la consolidación económica de cooperativas que han volcado su producción hacia el mercado exterior.

\section{1. - Cooperativas Exportadoras.}

Prácticamente la totalidad de las exportaciones de empresas cooperativas chilenas, se ubican bajo el concepto de "productos agroalimentarios". De acuerdo al Departamento de Cooperativas (2007), en el año 2005 fueron 14 las cooperativas chilenas que exportaron, al menos parte de su producción, totalizando US\$ FOB 30.340.583.-

En los años sucesivos, es decir 2006 y 2007, según la información de que dispone la Dirección de Promoción de Exportaciones ProChile ${ }^{6}$, son las siguientes cooperativas chilenas las que envían productos agroalimentarios al exterior:

\footnotetext{
${ }^{6}$ Por cierto, la información en cuestión es aquella en que es la propia empresa cooperativa la que exporta directamente, y no refleja los datos de las cooperativas que entregan a terceras empresas su producción para que ésta a su vez la exporte, así como tampoco los datos de producción de cooperativas, que es exportada a través de personas jurídicas distintas de la cooperativa.
} 
Cuadro N²: Cooperativas Chilenas Exportadoras, Años 2006 y 2007.

\begin{tabular}{|c|c|c|c|c|}
\hline \multirow[t]{2}{*}{ Nombre Cooperativa } & \multicolumn{2}{|c|}{2006} & \multicolumn{2}{|c|}{2007} \\
\hline & US\$ FOB & $\%$ & US\$ FOB & $\%$ \\
\hline $\begin{array}{l}\text { Cooperativa Agrícola y Lechera La Unión Ltda., } \\
\text { COLUN }\end{array}$ & 12.716.919.- & $52,7 \%$ & 25.721.594.- & $71,8 \%$ \\
\hline Cooperativa Agrícola Pisquera Elqui Ltda., CAPEL & 3.609.500.- & $14,9 \%$ & 1.063.970.- & $2,9 \%$ \\
\hline $\begin{array}{l}\text { Cooperativa Agrícola Vitivinícola de Curicó Ltda., } \\
\text { Viña Los Robles }\end{array}$ & 3.979.937.- & $16,5 \%$ & 5.385.311.- & $15,0 \%$ \\
\hline Cooperativa Agropecuaria Chiloé Ltda., CHILOLAC & 940.472.- & $3,9 \%$ & $0 .-^{7}$ & $0 \%$ \\
\hline $\begin{array}{l}\text { Cooperativa Campesina Apícola Valdivia Ltda., } \\
\text { APICOOP }\end{array}$ & 1.333.943.- & $5,5 \%$ & 1.567.775.- & $4,4 \%$ \\
\hline $\begin{array}{l}\text { Cooperativa Agrícola Vitivinícola de Cauquenes } \\
\text { Ltda., Viña Lomas de Cauquenes }\end{array}$ & 1.119.501.- & $4,6 \%$ & 1.411.769.- & $3,9 \%$ \\
\hline Cooperativa Campesina Convento Viejo Ltda. & 47.200.- & $0,2 \%$ & 19.250.- & $0,1 \%$ \\
\hline Cooperativa Campesina Entre Ríos Ltda. & 200.267.- & $0,8 \%$ & $0 .-$ & $0 \%$ \\
\hline Cooperativa de Trabajo Punta Chilen Ltda. & 21.600.- & $0,1 \%$ & $0 .-$ & $0 \%$ \\
\hline $\begin{array}{l}\text { Cooperativa Agrícola y Pisquera San Carlos Ltda., } \\
\text { OLIVIER }\end{array}$ & 1.970.- & $0,0 \%$ & $0 .-$ & $0 \%$ \\
\hline Cooperativa Agrícola Vitivinícola Loncomilla Ltda. & 43.200.- & $0,2 \%$ & 487.440.- & $1,4 \%$ \\
\hline Cooperativa Campesina Esperanza Ltda. & 120.463.- & $0,6 \%$ & 163.918.- & $0,5 \%$ \\
\hline
\end{tabular}

Fuente: Elaboración propia, a partir de Base de Datos de ProChile, Febrero de 2008.

A partir del Cuadro precedente, es posible relevar los siguientes aspectos:

- De las doce cooperativas que exportaban al menos parte de su producción en el año 2006, son sólo ocho las cooperativas que siguen exportando al año siguiente (2007)

- Las exportaciones de las cooperativas chilenas, aumentaron en US\$ FOB 11.686.055.-, entre el año 2006 y 2007. Representando un crecimiento del 48,4\%

- El año 2006, las tres cooperativas con mayores exportaciones (en US\$ FOB) concentraban un $84,1 \%$ de las exportaciones totales de cooperativos, mientras que el 2007, las tres principales alcanzan un $91,2 \%$

- La Cooperativa láctea COLUN, tiende a hegemonizar las exportaciones cooperativas chilenas, pasando de un 52,7 \% en el 2006, a un 71,8 \% en el año 2007.

\subsection{1. - Principales productos exportados.}

Respecto de los productos exportados por las cooperativas chilenas, para los años 2006 y 2007, éstos son:

a) Lácteos y derivados: queso (gouda, requesón), lactosuero, leche en polvo, leche y nata (crema), mantequilla (manteca), dulce de leche (manjar), edam, confites, chicles y otros en base a dulce de leche.

\footnotetext{
${ }^{7}$ En el transcurso del año 2007, CHILOLAC entra en una aguda crisis financiera que a la fecha, la tiene virtualmente quebrada.
} 
b) Vinos y pisco ${ }^{8}$ : carmenere, cabernet sauvignon, sauvignon blanc, syrah, merlot, chardonnay, otros vinos (blancos y tintos), mostos de uva, jugos de uva, aguardientes: de uva, de vino y de orujo de uvas.

c) Miel Natural.

d) Hortalizas: cebollas (frescas o refrigeradas), ajos (frescos o refrigerados),

e) Otros: confituras, jaleas y mermeladas, plantas, animales vivos.

A continuación se grafica la distribución porcentual del tipo de productos exportados por las cooperativas chilenas en el año 2007 (en base a los montos de US\$ FOB transados).

Gráfico N²: Productos Exportados por las Cooperativas Chilenas - Año 2007

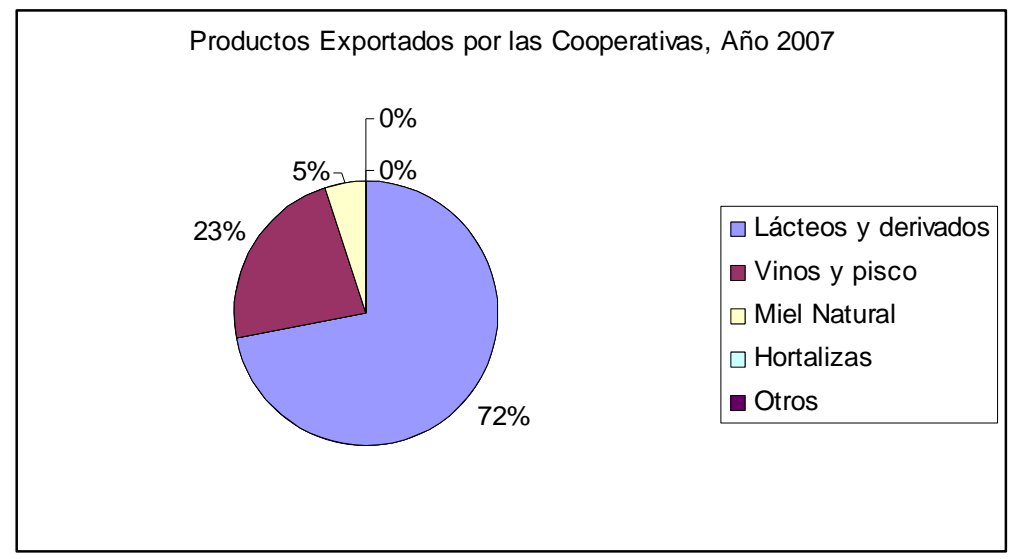

Fuente: Elaboración propia, a partir de Base de Datos de ProChile, Febrero 2008.

En el año 2007, aumenta aún más el peso relativo del rubro "lácteos y derivados" dentro de los productos exportados por las cooperativas nacionales, alcanzando un $72 \%$ del total (equivalente a US\$ FOB 25.721.594), comparado con el $57 \%$ que representaba en el año 2006. Los "vinos y piscos", se mantienen con un porcentaje importante (el $36 \%$ del 2006, disminuye a $23 \%$ en 2007), así como también la "miel natural" no sufre mayores cambios (pasando del $6 \%$ al 5, en el año 2007). Tanto "hortalizas", como "otros", tienden a desaparecer en el año 2007.

\subsection{2.- Países de Destino de las Exportaciones.}

Los principales países de destino de las exportaciones de las cooperativas chilenas, se presentan a continuación para el período 2007.

\footnotetext{
${ }^{8}$ Aguardiente de uva con denominación de origen, muy popular en Chile y Perú.
} 
Gráfico N 3: Países de Destino Exportaciones Cooperativas Chilenas - Año 2007

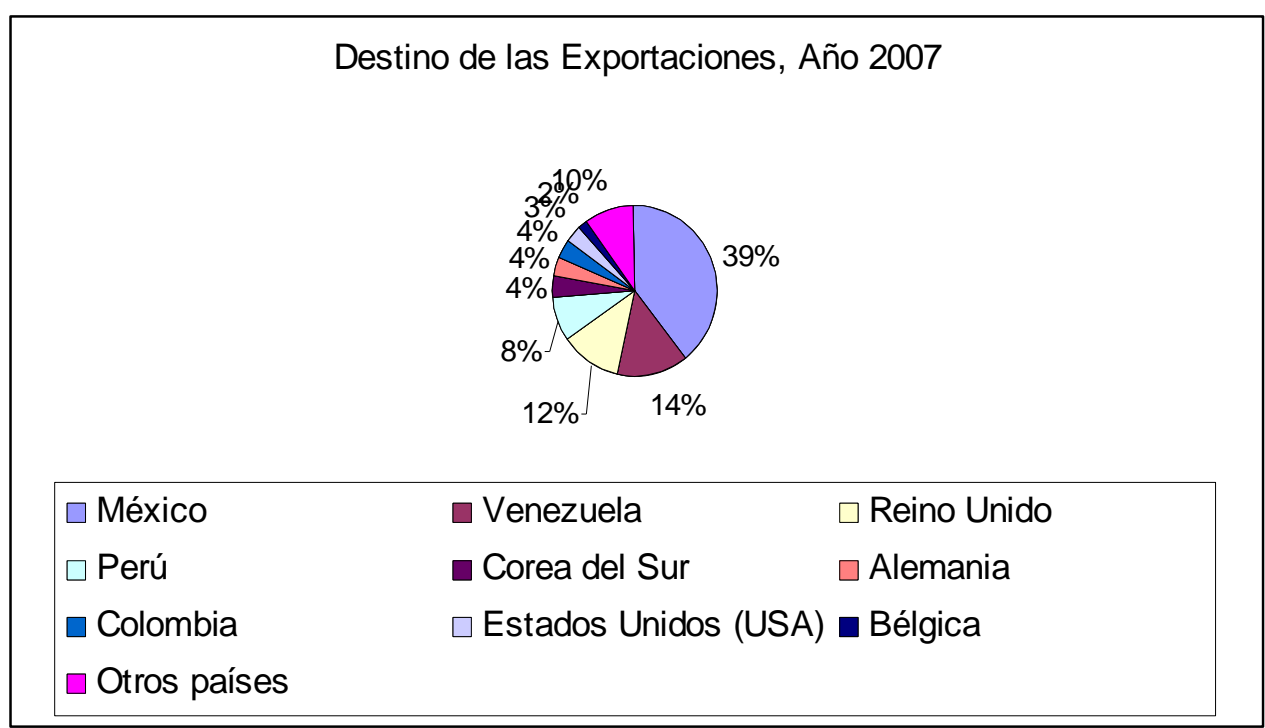

Fuente: Elaboración propia, a partir de Base de Datos de ProChile, Febrero de 2008.

En el año 2007, es México nuevamente el país que concentra la mayor parte del destino de las exportaciones de los productos agroalimentarios de las cooperativas chilenas, con 39 \% (para el año 2006, el porcentaje alcanzaba un $34 \%$ ). Luego se ubican, Venezuela, Reino Unido y Perú. Japón, que el año 2006 se $l l e v o$ un $10 \%$ de las exportaciones, ubicándose como el cuarto destino más frecuente, el año 2007 en cambio, sólo alcanza la ubicación 11ª. Colombia en cambio, se incorpora como destino preferencial, con un $4 \%$ de las exportaciones. Por otra parte, para el año 2007 los países de destino aumentan a 35 (versus 29 países, el año 2006), iniciándose intercambios comerciales con países como: Vietnam, Ucrania, República Checa, entre otros. Alemania, tal como en el año 2006, sigue siendo uno de los dos países con mayor intercambio comercial con cooperativas nacionales (cinco el año 2007, y el año anterior seis). Sin embargo, es ahora Francia, el país con el que mayor cantidad de cooperativas chilenas comercializan sus productos (seis).

\subsection{3.- Breve Análisis por Rubro.}

En este punto nos detendremos, en un breve análisis de los dos principales rubros donde se insertan las cooperativas chilenas en el mercado externo, a saber: "lácteos y derivados" y, "vinos y piscos".

a) Lácteos y derivados:

Es este un rubro con una importante presencia de cooperativas en Chile, agrupando a 2.503 productores que representan un $12 \%$ del total de productores nacionales, a través de ocho empresas cooperativas activas (Lobo, 2007). Dichas cooperativas han debido enfrentar en los últimos años, la apertura de Chile a través de la firma de tratados y otros acuerdos económicos, frente a grandes potencias lecheras como las vecinas Uruguay y Argentina, o Nueva Zelanda, entre otras. Mediante fuertes procesos de modernización y 
gracias a la gran implantación de las cooperativas en algunas regiones del país, han podido hacer frente a los embates del mercado internacional, manteniendo el control de la elaboración de un $20 \%$ de leche fluída, el $43 \%$ de la producción de quesos y un 36 \% de la producción de manjar (Departamento de Cooperativas, 2007 b).

En este contexto, la Cooperativa Agrícola y Lechera COLUN Ltda., ha conseguido situarse como la segunda empresa con mayor recepción de leche en Chile: “(...) primero está SOPROLE, luego COLUN y después NESTLÉ" (Eduardo Anríquez, Presidente de la Federación Nacional de Cooperativas Lecheras de Chile FENALECHE). ${ }^{9}$

A Enero de 2008, COLUN generaba empleo directo a 1.606 personas ( 1.418 hombres y 188 mujeres), y empleo indirecto a aproximadamente 5.000 personas. ${ }^{10}$ Por su parte, el Presidente de la Federación Nacional de Cooperativas Lecheras, destaca que: “(...) es la empresa lechera que paga los mejores precios durante el año y al final del año, paga adicionalmente un mes más de producción de leche, ósea que más principios cooperativos, dónde ?"

\section{b) Vinos y piscos:}

La industria vitivinícola chilena, en poco más de un década ha logrado posicionarse como uno de los rubros más dinámicos y en constante crecimiento (entre un 10 y $20 \%$ anual), dentro de las exportaciones nacionales (Lobo, 2007). Ello, en una estrategia de crecimiento basada en una buena relación precio - calidad ("best value", ó " value for money"). En este escenario, encontramos a tres importantes actores cooperativos: la Cooperativa Agrícola Vitivinícola de Curicó Ltda., Viña Los Robles; la Cooperativa Agrícola Vitivinícola de Cauquenes Ltda., Viña Lomas de Cauquenes; y, la Cooperativa Agrícola Vitivinícola Loncomilla Ltda. Todas ellas ubicadas en la zona centro sur del país, Región del Maule, agrupan a un total de 461 socios/ as (Departamento de Cooperativas, 2007 b).

La Cooperativa Loncomilla, a diferencia de las otras dos mencionadas, realiza un embotellado mínimo, orientando la mayor parte de su producción (aprox. 15 millones de litro) a la venta "a granel".

La Cooperativa Agrícola Vitivinícola de Curicó Ltda. (Viña Los Robles), por su parte, está constituida por 67 socios/ as, que suman aproximadamente 1.000 hectáreas. Cuarenta y dos de éstos socios, cuentan con menos de 10 hectáreas (Departamento de Cooperativas, 2007 b).

Finalmente, destacamos el caso de la Cooperativa Agrícola Vitivinícola de Cauquenes Ltda. (Viña Lomas de Cauquenes), conformada por 260 pequeños productores del secano interior de la Región del Maule, que precisamente por su ubicación en terrenos de "secano", deben enfrentar serias dificultades con el escaso rendimiento de sus predios. La Cooperativa fue en su momento, una de las primeras viñas en producir "vino orgánico", y es actualmente la única empresa vitivinícola chilena -y una de las tan sólo 35 a nivel mundial- certificadas en "comercio justo" (fair trade), por la Fairtrade Lavelling Organization (FLO).

En relación a la actividad pisquera, es necesario señalar que este sector tuvo un crecimiento sostenido de superficie hasta el año 1997, alcanzando a ocupar 10 mil hectáreas de plantaciones, localizadas en la región centro y norte de Chile. Históricamente, fueron dos cooperativas las que controlaban la industria, la Cooperativa

\footnotetext{
${ }^{9}$ Entrevista realizada en Noviembre de 2007.

${ }^{10}$ Información entregada por el Gerente de Administración y Finanzas de la Cooperativa (Enero de 2008), al Equipo de Investigación de Pro - Asocia, en el marco del Estudio "Impactos de la Integración Regional del MERCOSUR sobre el Sector Cooperativo". En el mismo Estudio, COLUN reporta que sus interlocutores extranjeros para la exportación son todas empresas privadas tradicionales -y no cooperativas-, así como también, que el 100 \% del financiamiento para exportar corresponde a "financiamiento propio".
} 
Agrícola Pisquera Elqui Ltda. (CAPEL), y la Cooperativa Agrícola Control Pisquero Elqui y Limarí Ltda. (CONTROL). En el año 2005, Ia Compañía Cervecerías Unidas CCU, absorbió la Cooperativa Control fusionando sus activos y marcas comerciales, a través de una nueva empresa (Compañía Pisquera de Chile), en la que cuenta con el $80 \%$ de participación (Lobo, 2007). A pesar de la gran arremetida de la nueva compañía, la Cooperativa CAPEL ha logrado mantener su liderazgo en la industria, gracias a un intenso proceso de modernización de su gestión y una creciente diversificación de su producción, que le permite sumar a sus tradicionales productos, la reciente elaboración y comercialización de cócteles tales como: caipiriña, piña colada, pisco con berries, entre otros.

\section{2. - Relaciones a Nivel Internacional de las Cooperativas Chilenas}

En el marco del Estudio "Impactos de la Integración Regional del MERCOSUR sobre el Sector Cooperativo" "11, el Programa Interdisciplinario de Estudios Asociativos de la Universidad de Chile, aplicó un cuestionario (encuesta auto-aplicada), a una muestra representativa de Cooperativas: de Trabajo (10), Agrarias (13) y de Ahorro y Crédito (17). Así entonces, el total de cooperativas encuestadas alcanza a 40, en la suma de los tres subsectores.

Frente a la pregunta: ¿ Su cooperativa, mantiene actualmente relaciones internacionales con alguna organización extranjera ?, los resultados son:

Gráfico N 4:

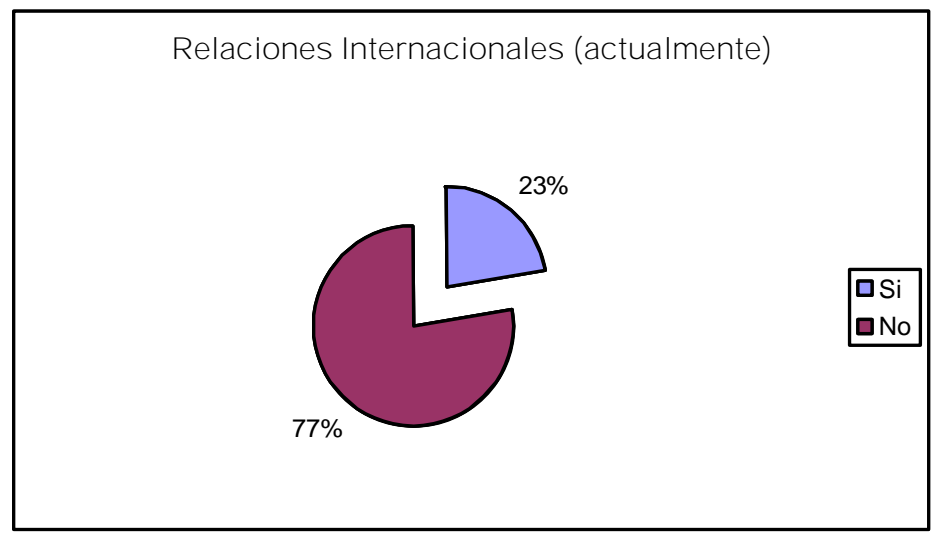

Fuente: Elaboración propia, a partir de Resultados Encuesta.

Al pedir caracterizar el tipo de realaciones con organizaciones extranjeras, éstas se concentran en el ámbito de la "asistencia técnica", por una parte, y por la otra, en las relaciones de cáracter comercial. En ambos casos, con un $29 \%$ La distribución total, se puede ver en el siguiente gráfico:

\footnotetext{
${ }^{11}$ Dicho Estudio, coordinado por la Universidad de la República (Uruguay) y con la participación de otras 4 Universidades de América Latina, cuenta con el patrocioinio del International Development Research Centre IDRC (Canadá).
} 
Gráfico N 5

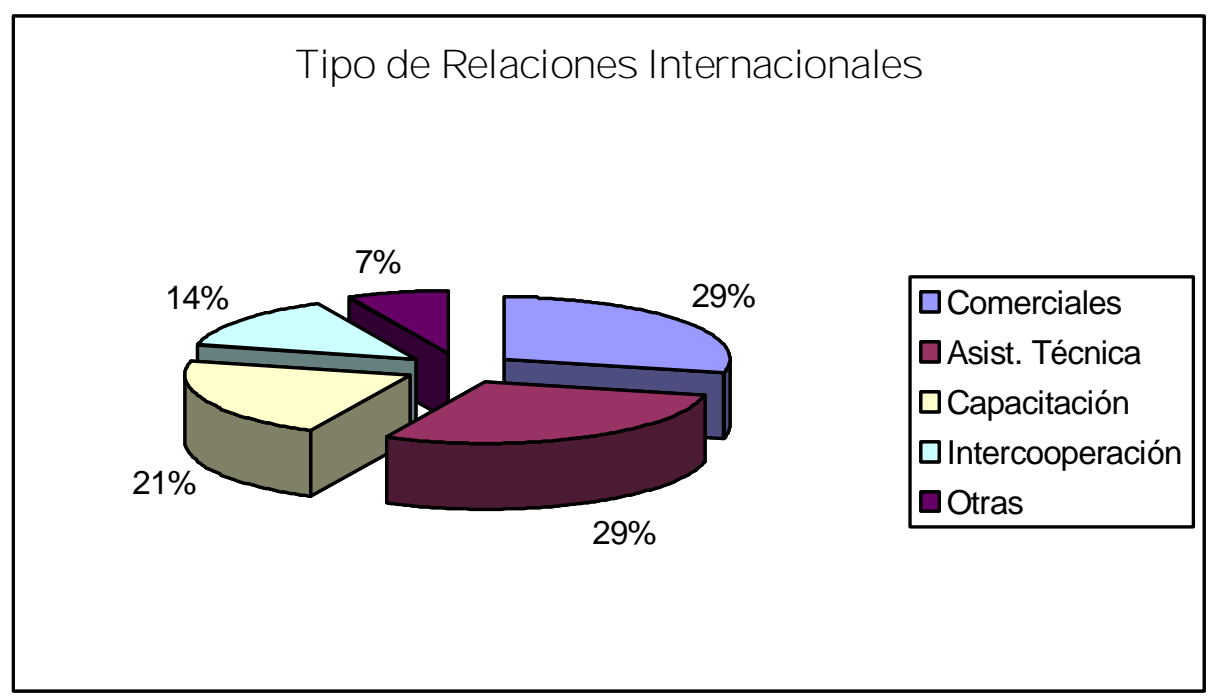

Fuente: Elaboración propia, a partir de Resultados Encuesta.

Respecto de las regiones del mundo, con las que se vinculan las cooperativas chilenas, los datos de la encuesta, indican la preeminencia de Europa, con un $40 \%$ del total, luego el Resto de América Latina (países no pertenecientes a MERCOSUR), con un 27 $\%$ de los casos, mientras MERCOSUR "ampliado" (Argentina, Brasil, Paraguay, Uruguay + Bolivia, Colombia, Ecuador, Perú y Venezuela).

Gráfico Nº

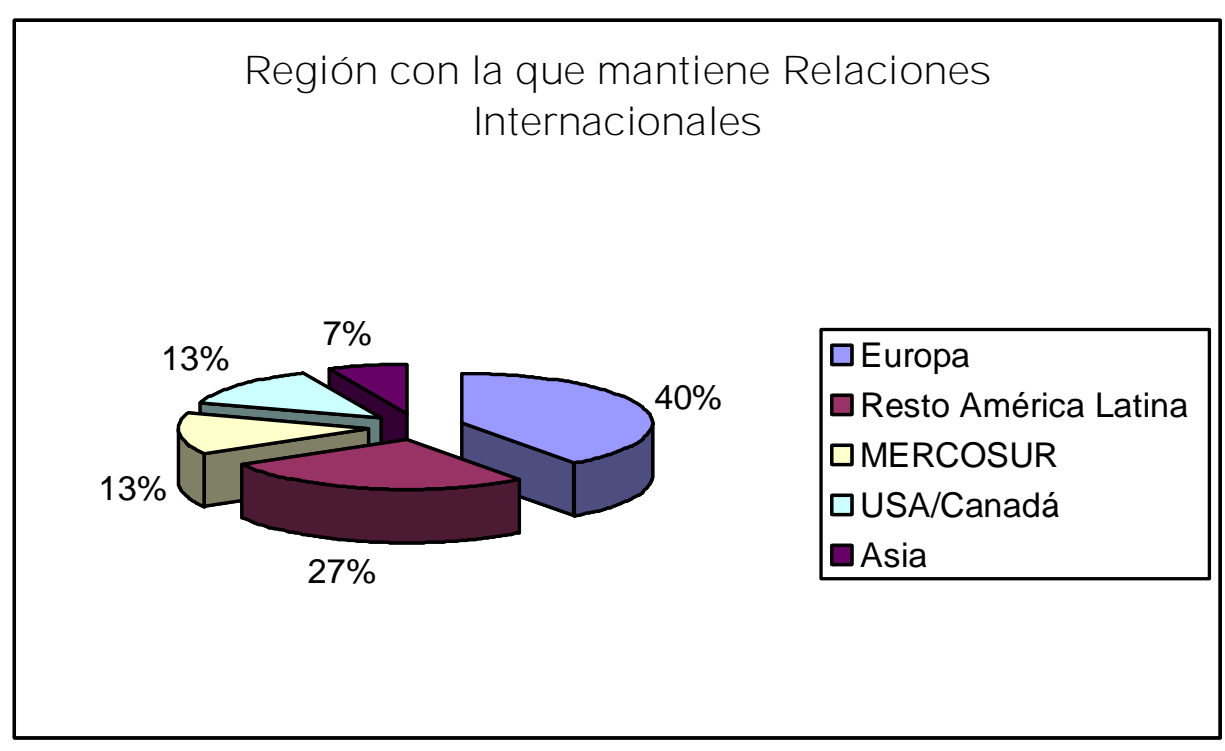

Fuente: Elaboración propia, a partir de Resultados Encuesta. 


\section{6.- ESTRATEGIAS DE INTERNACIONALIZACIÓN DE LAS COOPERATIVAS AGRARIAS CHILENAS}

Considerando los antecedentes entregados en los puntos anteriores, se pueden identificar diversas estratégias de internacionalización de lãs empresas cooperativas agrárias chilenas, que en general tienen uma relación directa com el rubro productivo específico em el cual se concentra la actividad de la cooperativa, más que con el temano de la empresa.

Es interesante destacar que especialmente para el caso de las cooperativas agrarias de menor tamaño, independientemente de su rubro, uno de las causas que explican la actividad a nivel internacional de la cooperativa tiene que ver con factores humanos, que tienen relación con el vínculo ya sea a nivel dirigencial o técnico de personas que tienen los contactos o vínculos a nivel internacional.

En todo caso es interesante y necesario destacar que del total de 259 cooperativas chilenas del sector agrícola, solamente 12 tienen actividades relacionadas con la exportación lo que corresponde solamente al $4,6 \%$ del total. Ahora bien, considerando los pocos casos disponibles, se pueden identificar tres tipos de estrategias desarrolladas por las cooperativas agrarias chilenas, em el campo de sus procesos de exportación:

\section{1.- La incorporación en redes de comercio justo de las cooperativas de menor tamaño}

Para las cooperativas de menor la principal estrategia de internacionalización a correspondido a la incorporación em diversas redes de comercio justo, que em determinados rubros productivos como la miel, el vino y la quinoa tienen um rol fundamental.

Para llevar adelante este proceso há sido habitual que a través del apoyo y asesoría técnica de uma ONG, Iãs cooperativas han podido certificar su producción, y de esta forma contar con la acreditación correspondiente para la comercialización a nível internacional de su producción.

Lo anterior ha tenido como consecuencia la posibilidad de contar con canales de comercialización relativamente seguros y poco competitivos, que si bien otorgan una cierta seguridad en la actividad comercial y productiva, restringe a la vez el tipo de canales de comercialización disponibles para la cooperativa.

\section{2. - La incorporación inestable en procesos de comercialización a nivel internacional}

Una segunda estrategia aplicada por las empresas cooperativas agrarias chilenas, ha correspondido a diversos procesos inestables en el tiempo de comercio a nivel internacional, que ha significado avanzar más bien por la vía del "ensayo y error", teniendo en la mayor parte de las ocasiones una imagen distorsionada de las beneficios reales de la actividad exportadora.

En este tipo de proceso es habitual la incorporación a algún programa público de promoción de exportaciones, que si bien no existen en particular para el sector cooperativo, si existen a nivel general, indistintamente del tipo de forma jurídica de la empresa. 
Con el apoyo de asesores externos, públicos o privados, se generan las condiciones para promover envíos del total o de una fracción de la producción de los asociados de la cooperativa, sin mayor claridad sobre el destino final de toda la operación.

Cuando la experiencia genera beneficios reales a los asociados, se hace viable la continuidad en el tiempo de la operación, pero muchas veces sujetos a las condiciones de las empresas intermediarias en procesos de comercio internacional, debilitando el margen de excedentes de las propias cooperativas, y sin posibilidad de que esta genere capacidades técnicas que permitan mayor control de todo el proceso.

\section{3.- La estrategia de las cooperativas agrarias con capacidad exportadora autónoma}

Esta estrategia se aplica a um número muy reducido de cooperativas, entre las cuales destaca la Cooperativa Lechera COLUN, que centrada en la exportación de productos lácteos y sus derivados, tiene una actividad internacional continua en el tiempo, y en base a canales de comercialización competitivos y a través de la aplicación de capacidades técnicas propias, muy difíciles de replicar por otras cooperativas nacionales.

\section{7.- $\quad$ CONCLUSIONES FINALES PRELIMINARES}

A continuación se presentan algunas conclusiones finales preliminares relativas al impacto de los procesos de globalización sobre el sector cooperativo chileno.

Alto impacto del proceso de globalización, aunque esta característica corresponde a la evaluación general del impacto sobre el sector cooperativo, y no necesariamente tiene que ver con la incorporación plena del sector cooperativo a procesos de internacionalización, ya sea participando en actividades de exportación o importación, sino que más bien al contexto general que afecta al sector cooperativo en el contexto de macados globalizados, propios de la actual situación de la economía chilena..

El sector cooperativo tiene una actividad internacional, en función de su actividad exportadora similar al sector empresarial chileno en general, pues si en el caso del sector empresarial chileno formal, para el año 2007 un total de 7.919 empresas realizaron actividades de exportación, representando así un 1,21\% del total de empresas chilenas, siendo para el caso cooperativo un total de doce empresas, lo que corresponde a un $1,3 \%$ del total de cooperativas activas.

Bajo impacto de los procesos de integración del MERCOSUR sobre el sector cooperativo, considerando que el sector cooperativo ha impulsado sus procesos de exportación considerando mercados muy amplios, en tres continentes distintos. En este punto la proximidad geográfica no ha significado un plus significativo.

Alta heterogeneidad y concentración de los volúmenes de exportación de los sectores cooperativos chilenos, lo que es coincidente con la situación global del sector exportador chileno. En este caso 3 grandes cooperativas concentran más del $80 \%$ del volumen de exportaciones, siendo también muy significativo que cooperativas de menor tamaño tienen dificultades significativas en mantener en el tiempo sus esfuerzos exportadores.

Esfuerzos "titánicos" de algunas cooperativas para insertarse en los procesos internacionales, especialmente aquellas de menor tamaño del sector agropecuario, casos 
en los cuales se realiza un esfuerzo que concentra la mayor cantidad de tiempo, energía y recursos de la empresa cooperativa, con resultados que a menudo son inciertos.

La inexistencia de programas públicos de apoyo a los esfuerzos de internacionalización del sector de empresas cooperativas, las cuales en el caso de querer explorar este tipo de actividades a nivel internacional, tienen que recurrir a los mecanismos generales de apoyo a la actividad exportadora, que por otra parte se concentra casi exclusivamente en las acciones que impulsa PROCHILE.

Efectos directos sobre las estructuras organizacionales de las cooperativas, que implican fuertes tensiones y reorganizaciones que muchas veces van más allá de las capacidades que las propias empresas cooperativas tienen a su alcance.

Importancia de las prácticas de comercio justo, en determinados casos las prácticas y mecanismos comerciales denominados de "comercio justo", han sido una vía de acceso de algunas cooperativas a mercados internacionales, que tienen esta condición particular que genera un identidad particular en mercados especializados.

Precariedades de los "soportes" organizacionales para enfrentar la globalización: idiomas, nuevas tecnologías, recursos financieros, lo que implica múltiples dificultades y contratiempos que generan lentitudes y exigencias para los cuales no se está preparado, y para los cuales no se cuentan con soportes públicos adecuados.

\section{BIBLIOGRAFÍA}

BARRÍA, Liliana (1990). Las Organizaciones de los Productores en la Articulación de la Agricultura y la Industria. Las Cooperativas Agroindustriales en Chile. Comisión Económica para América Latina y el Caribe CEPAL, Santiago de Chile.

CASTILLO, César; NARVARTE, Pedro y TORRES, Natalia (2006). Estudio de la Viabilidad Organizacional del Sector Cooperativo de Ahorro y Crédito en Chile. Ponencia presentada en 1er Coloquio Internacional de la Red RULESCOOP, Universidad de Bretagne Occidentale, Brest - Francia, 22 al 24 de Mayo de 2006.

CEPAL (1989). Cooperativismo Latinoamericano. Antecedentes y Perspectivas. Comisión Económica para América Latina y el Caribe CEPAL, Santiago de Chile.

DEL CAMPO, Pedro y RADRIGÁN, Mario (1998). El Sector Cooperativo Chileno: Tradición, Experiencias y Proyecciones. CONFECOOP - CCA, Santiago de Chile.

DEPARTAMENTO DE COOPERATIVAS, Ministerio de Economía (1992). El Sector Cooperativo en Chile. Departamento de Cooperativas del Ministerio de Economía, Fomento y Reconstrucción, Santiago de Chile.

DEPARTAMENTO DE COOPERATIVAS, Ministerio de Economía (2007 a). Estadísticas y Cifras del Sector Cooperativo. Departamento de Cooperativas del Ministerio de Economía, Fomento y Reconstrucción, Santiago de Chile.

DEPARTAMENTO DE COOPERATIVAS, Ministerio de Economía (2007 b). Panorama General del Sector Cooperativo Chileno. Unidad de Fomento, Capacitación y Estudios, Departamento de Cooperativas del Ministerio de Economía, Fomento y Reconstrucción, Santiago de Chile.

FRENCH-DAVIS, Ricardo (2002). El impacto de las exportaciones en el crecimiento de Chile. En: Revista de la CEPAL, Abril de 2002, № 76, Santiago de Chile. 
FURCHE G., Carlos (2001). Inserción de la agricultura chilena en los mercados internacionales. Documento de Trabaj o, Serie Comercio Exterior № 3, Oficina de Estudios y Políticas Agrarias ODEPA, Gobierno de Chile, Santiago de Chile.

HERNÁNDEZ A., Luis (2006). "Situación Actual del Cooperativismo en Chile." En: Impactos de la Integración Regional del MERCOSUR sobre el Sector Cooperativo. Cooperativas e Integración Regional MERCOSUR, con el apoyo de CIID (Canadá), Montevideo - Uruguay, Octubre de 2006. Págs. 79 - 83.

HERNÁNDEZ, Luis y RADRIGÁN, Mario (2006 c). El Cooperativismo en el Sector Agrícola Chileno. Trayectoria y Estrategias de Acción en el Contexto de la Globalización. Ponencia presentada en el IV Encuentro de Investigadores Latinoamericanos en Cooperativismo, Universidad Nacional de Rosario - Argentina, 14 - 15 de Septiembre de 2006.

LOBO G., Luis (2007). Cooperativismo Agropecuario: Una mirada desde la estrategia de desarrollo implementada por Chile. En: Trayectoria y Situación Actual de las Cooperativas en Chile: Trabajo, Agropecuarias, Ahorro y Crédito. Programa Interdisciplinario de Estudios Asociativos Pro - Asocia, Universidad de Chile: Santiago de Chile. Págs. 35 - 59.

MARTINI, Gabriela, PÉREZ, Ernesto y RADRIGÁN, Mario (2003). Situación Actual del Cooperativismo en Chile, Año 2002. Programa Interdisciplinario de Estudios Asociativos Pro - Asocia, Universidad de Chile, Santiago de Chile.

MATAMALA, Alexander (2004). Ineficiencia de la Ley 19.832, frente a la Igualdad Económica de las Cooperativas Agrícolas. Tesis de Grado, Universidad Austral de Chile, Valdivia - Chile.

WILLIAMSON C., Guillermo (1990). El Movimiento Cooperativista Campesino Chileno. Ediciones Universidad de la Frontera - PIIE, Santiago de Chile.

\section{ENDEREÇO DOS AUTORES}

Programa Interdisciplinario de Estudios Asociativos Pro - Asocia Universidad de Chile 